2002) edited by Lesley Rogers and Richard Andrews. Perhaps McManus opted not to include a lengthy discussion in his book to save space and to avoid distracting readers from the larger issues at hand.

Minor criticisms aside, he has done a marvellous job of summarizing and integrating a wide range of findings from various disciplines addressing questions on the nature of right and left. The presentation and clarity of the topics is palatable to both the scientific and lay communities, making this volume well worth reading.

William D. Hopkins is in the Division of

Psychobiology, Yerkes National Primate Research Center, 954 Gatewood Road, Atlanta, Georgia 30322, USA.

\section{Controlling a generous host}

\section{Parasites and the Behavior of} Animals

by Janice Moore

Oxford University Press: 2002. 338 pp. $\mathfrak{E 6 5}$, $\$ 85$ ( hbk); £24.95, \$45 (pbk)

\section{Paul Schmid-Hempel}

In the early 1980s, a paper in Scientific American explained that a pill-bug (or woodlouse) is not always a pill-bug because its behaviour may be dramatically changed by an internal parasite to suit the latter's selfish interests. Although this was not the first summary of such parasite-induced alterations of host behaviour, it arrived in a climate of a suddenly increased awareness of the role of parasites in host ecology and evolution. Since then, the field as a whole has witnessed an unprecedented increase in the number of studies and publications.

Contrary to earlier beliefs, research over the past two decades has shown that parasites are not the exotic case nor a dull, degenerate lot that seek a harmonious coexistence with their hosts. Rather, parasites are ubiquitous and are continuously, relentlessly coevolving to overcome their hosts' defence systems. Parasite strategies, too, are governed by short-term fitness maximization even if it means that the host is not always killed or made sick. In doing so, parasites face the central dilemma of inflicting damage to the current host while still needing transmission to the next - a problem elegantly captured in Roy Anderson and Robert May's classical equation for $R_{0}$, the parasite's net reproductive rate.

Even though we are still far from a comprehensive understanding, the application of such population and evolutionary thinking to host-parasite interactions is a success story. Strangely, as the field progressed, investigations into how and why parasites

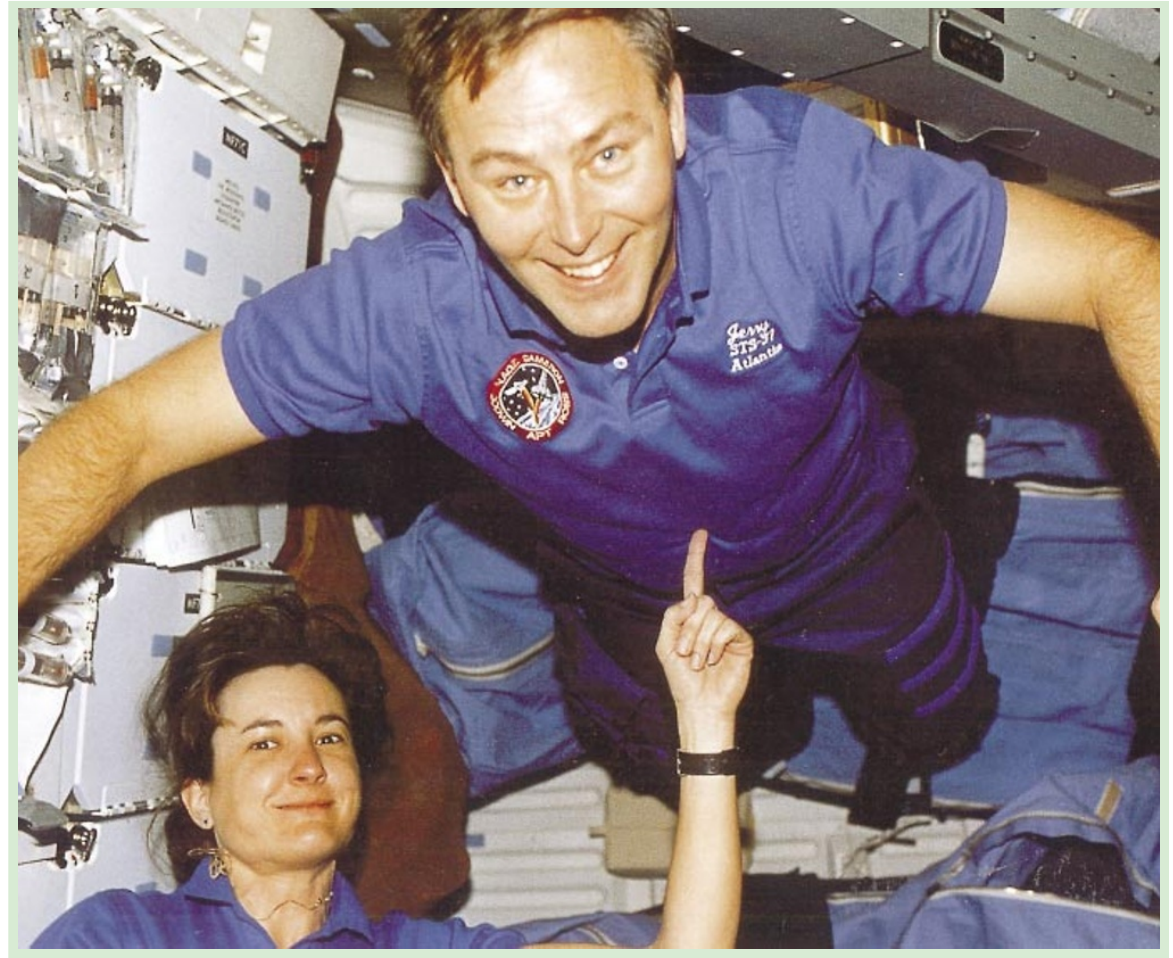

\title{
Tripping the light fantastic
}

In the 40-odd years since Yuri Gagarin was the first man in space, some 400 others — including Linda Godwin (left) and Jerry Ross, shown above in the weightless conditions on board Atlantis -

affect host behaviour fell behind. Janice Moore, the author of the Scientific American paper on pill-bugs, now sets out to correct this imbalance.

Her book is a gripping account of the sometimes spectacular behavioural (and morphological) alterations caused by parasites. She concentrates on infections by helminths ('worms'), such as nematodes, cestodes and trematodes, but regularly discusses fungi and protozoa too. Did you know that the first experimental demonstration of how tapeworms infect humans was made with the help of the guillotine? (See p. 20 of the book if you want the gruesome details).

Moore's writing is witty and conveys the flavour of her deep interest in beasts that most people like to avoid. Her organizing principle, which unfortunately is not carried through consistently, is the equation for $R_{0}$. In fact, altered host behaviour directly affects most of the equation's variables. For example, many parasites render the host fearless. This increases the probability that the current host will be eaten by a predator that may then serve as the next host. Conversely, host avoidance behaviour reduces the risk of infection. Both processes change the numerical value of the transmission rate in the basic equation. Moore does not restrict herself to simple behaviours, however, but sensibly includes changes in life-history parameters, too. For example, have made the same remarkable journey. Serge Brunier looks back at the highs and lows in Space Odyssey: The First Forty Years of Space Exploration (Cambridge University Press, £25).

behavioural fever and behavioural chill, in response to infection, each reduce mortality rates and may accelerate recovery.

For the population biologist, behavioural alterations count if the parameters of the equations vary. Behaviourists like to trust their interpretation of the meaning of an observed behaviour. Physiologists want to know how parasites achieve their manipulative tricks. Moore's treatise gives all sides something to chew on, but it does not generate many new concepts to fill the gaps.

To pick a few faults, figures are not always attractive or well explained, quotations are occasionally unfortunate, and there are some unnecessary repetitions. But these minor shortcomings aside, the book is a pleasant read (even though some examples can send chills up your spine), a highly stimulating survey of the field, and certainly a reference for years to come. Everyone with a general interest in biology who can still be amazed by the awesome power of natural selection should look at this book. Along the way, you will learn many things and perhaps come up with a question that might change your own research. In this sense, the book is an important step. Hopefully, the next such step is not another 20 years away.

Paul Schmid-Hempel is in the Department of Environmental Sciences, ETH Zurich, CH-8092 Zurich, Switzerland. 